OPEN ACCESS

Edited by:

Ute Frevert

New York University School of

Medicine, USA

Reviewed by:

Giampietro Corradin,

University of Lausanne, Switzerland

Liisa Kaarina Selin,

University of Massachusetts Medical

School, USA

Christine Benn,

Statens Serum Institut, Denmark

${ }^{*}$ Correspondence:

Eric Muraille

emuraille@hotmail.com

Specialty section:

This article was submitted to

Microbial Immunology,

a section of the journal

Frontiers in Microbiology

Received: 20 August 2015 Accepted: 18 December 2015

Published: 11 January 2016

Citation:

Muraille E (2016) The Unspecific Side of Acquired Immunity Against

Infectious Disease: Causes and Consequences

Front. Microbiol. 6:1525. doi: 10.3389/fmicb.2015.01525

\section{The Unspecific Side of Acquired Immunity Against Infectious Disease: Causes and Consequences}

\author{
Eric Muraille * \\ Laboratoire de Parasitologie, Faculté de Médecine, Université Libre de Bruxelles, Bruxelles, Belgium
}

Acquired immunity against infectious disease (AlID) has long been considered as strictly dependent on the $B$ and $T$ lymphocytes of the adaptive immune system. Consequently, AllD has been viewed as highly specific to the antigens expressed by pathogens. However, a growing body of data motivates revision of this central paradigm of immunology. Unrelated past infection, vaccination, and chronic infection have been found to induce cross-protection against numerous pathogens. These observations can be partially explained by the poly-specificity of antigenic $T$ and $B$ receptors, the Mackaness effect and trained immunity. In addition, numerous studies highlight the importance of microbiota composition on resistance to infectious disease via direct competition or modulation of the immune response. All of these data support the idea that a non-negligible part of AlID in nature can be nonspecific to the pathogens encountered and even of the antigens expressed by pathogens. As this protection may be dependent on the private $T$ and $B$ repertoires produced by the random rearrangement of genes, past immune history, chronic infection, and microbiota composition, it is largely unpredictable at the individual level. However, we can reasonably expect that a better understanding of the underlying mechanisms will allow us to statistically predict cross-protection at the population level. From an evolutionary perspective, selection of immune mechanisms allowing for partially nonspecific AlID would appear to be advantageous against highly polymorphic and rapidly evolving pathogens. This new emerging paradigm may have several important consequences on our understanding of individual infectious disease susceptibility and our conception of tolerance, vaccination and therapeutic strategies against infection and cancer. It also underscores the importance of viewing the microbiota and persisting infectious agents as integral parts of the immune system.

Keywords: infectious diseases, microbiota, acquired immunity, cross-protection, heterologous immunity, mackaness effect, trained immunity, nonspecific effects of vaccines

\section{ACQUIRED IMMUNITY AGAINST INFECTIOUS DISEASE}

Long ago, physicians observed that people who had recovered from the plague never got it again and displayed "acquired immunity" against infectious disease (AIID). Early vaccination techniques developed by Jenner were based on these observations and immunology stemmed, in large part, from the necessity to understand how vaccines work in order to improve their efficacy and their safety. For decades, AIID has been explained to immunology students as strictly 
dependent on the adaptive immune system composed of B and $\mathrm{T}$ lymphocytes expressing highly specific antigenic receptors. As a result, the development of vaccines has been closely linked to the identification of immunodominant antigens expressed by pathogens, with vaccination being presented as an infectious disease-specific intervention. However, a growing body of data from epidemiology, vaccinology, and experimental immunology research supports the idea that AIID can also be frequently nonspecific to the antigens expressed by pathogens. Depending on the case, this phenomenon has been explained by (i) cross-protection induced by vaccination or past infection, (ii) the "Mackaness effect" resulting from unrelated chronic infection, and (iii) microbiota-mediated protection.

\section{Nonspecific Protective Effects of Vaccination}

Numerous epidemiological and experimental studies have reported that previous exposure to unrelated infectious agents can greatly alter the host's immune response to an infection and induce a state of protective immunity, frequently termed "heterologous immunity" (reviewed in Welsh and Selin, 2002; Selin et al., 2011; Benn et al., 2013; Aaby et al., 2014). For example, epidemiological studies have shown that individuals vaccinated with vaccinia virus were less susceptible to infectious diseases such as measles, scarlet fever, whooping cough, and syphilis when compared to non-vaccinated persons (Mayr, 2004). In experimental studies, BCG vaccination protected not only against mycobacteria, but also against secondary infections with Listeria monocytogenes (Blanden et al., 1969), Salmonella typhimurium (Blanden et al., 1969), Staphylococcus aureus (Sher et al., 1975), Candida albicans (Sher et al., 1975; Van't Wout et al., 1992), Plasmodium yoelii (Matsumoto et al., 2000; Parra et al., 2013), and Schistosoma mansoni (Tribouley et al., 1978). Of course, nonspecific vaccine effects are not always positive. For example, while the diphtheria-tetanus-pertussis (DTP) vaccine protects against the three targeted diseases, it has been documented to increase female mortality from other infectious diseases (Aaby et al., 2012). The mechanisms underlying this negative effect are still unknown and remain to be investigated. On the whole, these data suggest that, in addition to their antigen- or target-specific effects, live vaccines "have nonspecific effects that may be just as important, or even more important" (Benn et al., 2013). As expected, similar experimental observations have been made with non-attenuated, fully virulent pathogens. For example, memory cytotoxic CD ${ }^{+}$ $\mathrm{T}$ lymphocytes induced by lymphocytic choriomeningitis virus (LCMV) infection are reactivated by the Pichinde virus and vaccinia virus, demonstrating that prior immunity to a specific virus could modulate future primary immune responses to a second, unrelated virus (Selin et al., 1994). We should also note that a comparison of homologous and heterologous protection showed that heterologous protection was generally considerably less than the almost total protection seen after challenge with a homologous infectious agent (Frenkel and Caldwell, 1975).

\section{Chronic Infection and the "Mackaness Effect"}

Research has long neglected the study of "slow infection" (associated with a long incubation period) and chronic infection by stealth or silent pathogens, such as herpesviruses, cytomegalovirus, Brucella, and Bartonella, which are the causative agents of several under-diagnosed chronic diseases constituting the most common infectious diseases. However, it has now become clear that persistence of a pathogen, even at very low levels, can affect the ability of the immune system to react to a new unrelated infection (reviewed in Stelekati and Wherry, 2012). Chronic infection can reduce or enhance the ability to control unrelated pathogens, a phenomenon termed the "Mackaness effect" in reference to the seminal work of Mackaness (Mackaness, 1964; Blanden et al., 1969) in 1964 demonstrating cross-protection between L. monocytogenes, Brucella abortus and Mycobacterium tuberculosis in mice (see Figure 1). More recent studies showed that herpes virus infection can confer beneficial protection against L. monocytogenes and Yersinia pestis (Barton et al., 2007), Helicobacter pylori infection is associated with protection against tuberculosis (Perry et al., 2010) and cytomegalovirus infection enhances the immune response to influenza vaccination (Furman et al., 2015). The precise molecular mechanisms involved in the Mackaness effect have yet to be elucidated. However, some aspects of the innate immune response, such as polarization of macrophages (Potian et al., 2011), stable histone changes in innate immune effector cells (epigenetic reprogramming) (Kleinnijenhuis et al., 2012) and nutritional immunity (Nairz et al., 2010; Cassat and Skaar, 2013), may account for the protective effect of chronic infection.

\section{Microbiota-Mediated Protection Against Infection}

Microbes colonize mammalian hosts immediately after birth, forming a resident-specific microbial community including bacteria, fungi, and viruses. This is referred to as the "microbiota." Numerous authors (Kitano and Oda, 2006; CerfBensussan and Gaboriau-Routhiau, 2010) have proposed that the microbiota can be considered as an integral part of the immune system. In support of this view, it is well known that germ-free mice are highly susceptible to infection (reviewed in Cerf-Bensussan and Gaboriau-Routhiau, 2010) and that the microbiota is required for adequate immune system maturation (Ivanov et al., 2009; Buffie and Pamer, 2013) and can contribute to the control of infectious microorganisms (Ivanov et al., 2009; Buffie and Pamer, 2013; Kamada et al., 2013). How the microbiota prevents pathogen colonization has been studied for many years, and the mechanisms involved largely fall into two categories: direct interactions between the microbiota and pathogens (competition-mediated colonization protection) and microbiota-mediated enhancement of host defense mechanisms (immune-mediated colonization protection). The microbiota can compete with pathogens to acquire nutrients, produce toxic compounds such as $\mathrm{H}_{2} \mathrm{O}_{2}$ or target structures that mediate adherence to the epithelium of the competing microorganism (reviewed in Bosch et al., 2013). The microbiota can also affect the 


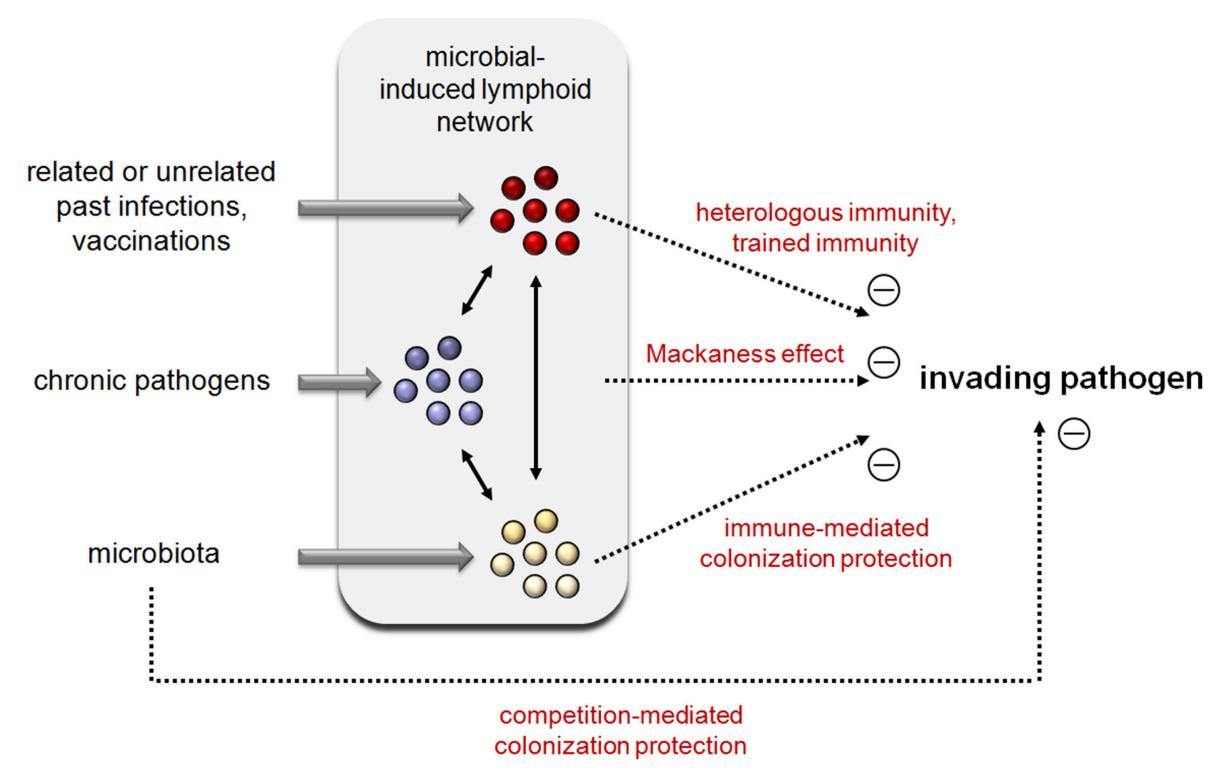

FIGURE 1 | Integrated view of the impact of past infection, chronic pathogen infection, and the microbiota on new invading pathogens.

responsiveness of the innate immune system (Wang et al., 2013), induce a cross-reacting immune repertoire able to recognize pathogens (reviewed in Buffie and Pamer, 2013) and favor the persistence of peripheral memory T cells (Tanaka et al., 2007), probably via cross-reactivity between the antigens recognized by memory $\mathrm{T}$ cells and the variety of antigens derived from the members of the microbiota. Systematic studies of the dynamic interactions between the microbiota and pathogens at the host mucosal surface could help develop predictive models of crossprotection (Bosch et al., 2013).

\section{THE NONSPECIFIC SIDE OF THE ADAPTIVE IMMUNE RESPONSE}

All of the data discussed above indicate that the efficiency of the individual immune response to fight infectious microorganisms is largely dependent on endogenous (mutualistic and parasitic) microorganisms and the past immune history of the host (Figure 1). Recent discoveries regarding the poly-specificity of antigenic receptors, the important role of innate immune cells in regulation of the adaptive response and trained immunity have provided a better understanding of the underlying mechanisms of cross-protection.

\section{The "Poly-Specificity" of B and T Lymphocytes}

The "clonal selection hypothesis" developed by Jerne in 1954, which is based on the "Lock and Key" model of Emil Fischer, proposes that each lymphocyte bears a single type of antigen receptor with unique specificity (the "one clonotypeone specificity" paradigm). Under that theory, stimulation of this receptor is required for activation of the cell and entrance of an antigen into the body results in the selection of only a few B lymphocytes that recognize the antigen and produce a corresponding antibody to destroy it. However, since this pioneering time, the "poly-specificity" (also termed polyreactivity, plasticity or degeneracy) of B cell receptor (BCR) and $\mathrm{T}$ cell receptor (TCR) [functionally the ability of a single receptor to specifically recognize many different antigens, as discussed in (Wucherpfennig et al., 2007)] has been well documented [reviewed in (Notkins, 2004) for BCRs and in (Wucherpfennig, 2004; Eisen and Chakraborty, 2010; Sewell, 2012) for TCRs]. Poly-specificity of the BCR has been mostly observed with natural antibodies, such as IgM in particular, that dominate the newborn B cell repertoire (Chen et al., 1998). Poly-specific BCRs bind to self-antigens as well as to a variety of bacteria and viruses (Oldstone, 2014), suggesting that they may be involved in preventing the dissemination of pathogens. Their biological importance has been demonstrated in antibody-deficient mice, which can be protected against lethal infections via the transfer of serum from antibody-competent naive animals (Ochsenbein et al., 1999; Jayasekera et al., 2007; Zola et al., 2009). Concerning the TCR, it has been estimated, for example, that a single patientderived autoimmune CD8 $\mathrm{T}$ cell clone recognizes over one million distinct decamer peptides in the context of a single $\mathrm{MHC}$ class I molecule (Wooldridge et al., 2012). As discussed by the authors, the poly-specificity of TCRs appears to be crucial for the interaction of an individual's limited TCR repertoire $\left(<10^{8}\right.$ $\mathrm{T}$ cell clones) with the $>10^{15}$ peptide MHC constituting the vast array of different potential ligands. Experiments have shown that peptides do not necessarily need to show high sequence homology to cross-react with the same T cell (Joshi et al., 2001). Consequently, even the injection of a specific (purified and well defined) antigen can induce the activation and expansion of a lymphocyte population able to potentially interact with more 
than just the antigen itself. As the reactivity of this population is not easy to predict, it is no longer appropriate to use the term "specific" (in the meaning of "definite" or "precise") to describe the immune response induced by a specific antigen. In addition, the physiological state of memory $\mathrm{T}$ cells is such that they are primed for activation, and they have been observed to be productively stimulated by a peptide concentration that is 50 times lower than the one required for the stimulation of naive T cells (Curtsinger et al., 1998). So, it would not be surprising if a memory $\mathrm{T}$ cell could be stimulated by a cross-reactive peptide with substantially lower affinity for the TCR than the "original" peptide that created the memory T-cell pool. A theoretical study suggests that although cross-reactivity is a rare event for immunologically naive individuals, the probability of crossreactive memory $\mathrm{T}$ cells becomes very high following successive infections (Zarnitsyna et al., 2013). B and T lymphocytes also express a great number of pattern recognition receptors (PRRs), such as Toll-like receptors (TLRs), that are able to interact with microbial associated structures or stress-induced molecules and induce or modulate their activation (Rawlings et al., 2012). Polyclonal stimulation by a microbial product such as CpG DNA may participate in the long-term maintenance of memory B cells (Bernasconi et al., 2002). Bystander infections can also promote optimal memory $\mathrm{T}$ cell responses by providing inflammatory signals such as IL-15 that are critical for the rapid proliferation of memory CD8 ${ }^{+} \mathrm{T}$ cells (Richer et al., 2015). A recent study showed that inflammatory monocyte-derived IL-15 and IL-18 can activate memory $\mathrm{CD}^{+} \mathrm{T}$ cells in a bystander manner in the apparent absence of TCR ligation (Soudja et al., 2012). In summary, an accumulating mass of evidence demonstrates that lymphocyte activation via antigen receptors may also not be "exquisitely antigen-specific" (Alberts et al., 2002) as previously considered and could furthermore be dependent on nonspecific innate immune receptors.

\section{Orchestration of the Immune Response}

The metaphor by which lymphocytes act as a conductor orchestrating the immune response has been used widely. This idea progressively changed at the end of the twentieth century when immunologists fully realized the crucial role of nonspecific innate immunity in regulating lymphocyte activity and the immune response. In fact, a growing body of data suggests that stromal cells, innate immune cells and "non-conventional" lymphocytes, such as $\gamma \delta \mathrm{T}$ cells, natural killer T (NKT) cells, and $\mathrm{B} 1$ lymphocytes, play an important role in regulation of both the primary and memory immune response to infectious agents (reviewed in Degli-Esposti and Smyth, 2005; Mueller and Germain, 2009; Bonneville et al., 2010). These non-conventional lymphocytes usually display low specificity and frequently appear to be pre-selected to recognize pathogen-associated molecular patterns (PAMPs). $\gamma \delta \mathrm{T}$ cells recognize a plethora of factors induced by stress conditions, few of which are characterized (Bonneville et al., 2010). NKT cells are activated by lipoprotein PAMPs presented by CD1 receptors (Rossjohn et al., 2012). B1 lymphocytes are frequently poly-specifics and seem to be selected to recognize both self-antigens and PAMPs (Baumgarth, 2011).

\section{Trained Immunity}

Until less than a decade ago, there was a general assumption that the $\mathrm{B}$ and $\mathrm{T}$ lymphocytes of the adaptive immune system were the only components able to generate memory cells, and mount recall memory responses (Murphy et al., 2008). Several recent studies have challenged this dogma (reviewed in Netea et al., 2011; Min-Oo et al., 2013; Martin, 2014), suggesting that the innate immune system displays adaptive properties. Natural Killer (NK) cells (that do not express specific antigen receptors) can autonomously retain a memory of past antigen encounters and mediate more robust secondary responses (Cooper et al., 2009). In some cases, protection has been associated with the clonal expansion of NK cells (Keppel et al., 2013). Until now, it has not been clear whether the functional antigen specificity of NK memory is comparable to that of $\mathrm{T}$ and B lymphocyte-mediated memory. Several studies also provide evidence that monocytes exposed in vivo to pathogens (Quintin et al., 2012) mount protective recall responses to re-infection, suggesting that even cells derived from the myeloid lineage in mammals may possess features of adaptive immunity. This phenomenon seems to be linked with epigenetic modifications, as stable histone methylation changes have been observed in the genome of innate memory cells (Quintin et al., 2012). The term "trained immunity" has been proposed (Netea et al., 2011) for the persistent enhanced state of the innate immune response following exposure to certain infectious agents, which may result in increased resistance to related or unrelated pathogens. As expected, a part of the cross-protection induced by vaccines seems to be dependent on trained immunity (reviewed in Blok et al., 2015). Interestingly, although only recently described, this phenomenon seems to be very common among living organisms. Plants, which possess only an innate immune system, also display an enhanced state of resistance and faster and stronger responses following infection (Fu and Dong, 2013). The underlying mechanisms seem also to include epigenetic modifications.

The mechanisms explaining cross-protective immunity are not limited to poly-specificity of lymphocytes, the Mackaness reaction, trained immunity, or microbiota-mediated protection. Infection can durably remodel mucosal tissues, thus favoring or impairing immune responses against unrelated pathogens (reviewed in Didierlaurent et al., 2007; Foo and Phipps, 2010). For example, in the lung, successive infections modify epithelium adherence and change the lymphatic network and the frequency of inducible bronchus-associated lymphoid tissue (iBALT). In addition, it would be wise to consider each infection as a unique phenomenon, as each pathogen displays particular immune escape mechanisms directly affecting the immune system. For example, a recent study (Mina et al., 2015) has demonstrated that a large part of nonspecific measles vaccine benefits come from the ability of the vaccine to impair measles-induced immune suppression characterized by systemic depletion of lymphocytes and reduced innate immune cell proliferation. By preventing measles-associated immune memory loss, measles vaccination also induces polymicrobial herd immunity. 


\section{A NEW COMPLEX ECOLOGICAL PARADIGM OF IMMUNITY EMERGES}

\section{The Immune System: A Nonlinear, Interactive, and Evolving Network}

Viewed as a whole, previous data support the idea that a non-negligible part of AIID is nonspecific to the antigens expressed by pathogens and is dependent on the past and present interactions of the host immune system with its environment. The ecological vision of the host protective immune response as dependent on multiple environmental factors is not new. In 1966, Fazekas de St Groth et al. already wrote that "Response to vaccine depends not only on the nature of the antigen itself but also on the immunological history of the recipient" and introduced the phenomenon of "original antigenic sin" to describe the inability of an experimented immune system to produce antibodies in response to a new antigen (Fazekas de St Groth Webster, 1966). In the same way, the "hygiene hypothesis" was proposed by Strachan (1989) to explain the rapid rise of asthma during the twentieth century. The original formulation of the hygiene hypothesis states that a lower incidence of infection in early childhood could affect the maturation of immune responses and favor the development of allergic diseases. During the last decade, this hypothesis has been progressively extended (Oikonomopoulou et al., 2013) to the development of autoimmune diseases and cancer, popularizing the idea that immune responses against pathogens, allergens, or tumors can influence each other nonlinearly. If we accept that immune cross-protection is more common in nature than previously expected, we can no longer look at vaccination or infection events independently of each other. Experimental and epidemiologic studies on cross-protection suggest that memory lymphocytes do not form fixed and isolated clusters of cells but rather an interactive and evolving cell nonlinear network displaying the properties of "adaptive complex systems” (CASs) (Holland, 2006; Brownlee, 2007) described in systems biology. CASs are open and dynamic nonlinear networks of interdependent agents, constantly acting and reacting to what the other agents are doing and to forces external to the system. As a result of these permanent complex interactions, CASs exist in a state of dynamic flux and change constantly and discontinuously. Antigenic challenge can alter the reactivity of the immune network by modifying the frequency of $\mathrm{T}$ and $\mathrm{B}$ lymphocytes and their polarization. The trained immunity phenomenon suggests that the innate immune system can also participate in this network, memorize past experiments and durably affect the polarization and reactivity of lymphocytes.

\section{The Microbiota and Pathogens form Integral Parts of the Host Immune System}

Mounting evidence supports the view that eukaryotic hosts and their microbiota have co-evolved toward mutualistic interactions benefiting each partner. This vision suggests that the microbiota and the host form a superorganism (Eberl, 2010) (also termed metaorganism; Bosch and McFall-Ngai, 2011) where the immune system plays not a "military role" (fighting well defined enemies) but a "police role" (penalizing parasitic/selfish comportments) to shape homeostasis within this consortium (Muraille, 2013). As previously discussed, the microbiota plays undeniable direct and indirect roles in the control of infectious microorganisms (Ivanov et al., 2009; Buffie and Pamer, 2013; Kamada et al., 2013). However, the distinction between the microbiota and pathogens is artificial and confusing as colonization by "pathogenic" bacteria or viral species is very frequent under healthy circumstances. For example, Streptococcus pneumoniae, $H$. influenzae, and $S$. aureus are commonly found in the respiratory tract of healthy individuals (reviewed in Bosch et al., 2013) and are considered as pathobionts (i.e., potentially pathological organisms which, under normal circumstances, live like commensals). In several infectious models (Belkaid et al., 2002; Obar et al., 2006; Beura et al., 2015), persisting pathogens have been described to enhance $\mathrm{T}$ cell memory and assure protection against secondary infection (via the concomitant immunity phenomenon). By inducing a cross-reactive repertoire, the Mackaness reaction or trained immunity, the pathogens can frequently participate in protection of the host against unrelated infectious diseases. These observations have prompted me to propose that the concept of the microbiota as forming an integral part of the host immune system (Kitano and Oda, 2006; CerfBensussan and Gaboriau-Routhiau, 2010) should logically be extended to pathogens. The host, its specific microbiota and the infecting microrganisms are interdependent and form a highly interactive network. Considering each part independently of the others is a reductionist view that has led us to neglect numerous important natural phenomena such as nonspecific AIID.

Some immunologists are still reluctant to admit the possibility of a nonspecific side of AIID. All immunological textbooks cite "specificity" as a hallmark of the adaptive immune response. In fact, this paradigm derives mainly from the use of simple proteins, such as ovalbumin, to study adaptive response. The administration of simple proteins in an inbred naive mice model induces a highly reproducible, and thus predictable, immune response. Retrospectively, this approach, through successful, appears to be questionable. First of all, this type of antigen does not naturally induce an immune response. Single proteins generally need to be associated with an adjuvant, the "immunologist's dirty little secret" as termed by Janeway (1989), to induce the inflammatory reaction required for lymphocyte activation. Under natural conditions, the reaction to a simple, non-proliferating, and non-damaging molecule is a trait of an allergic or autoimmune reaction and is generally not associated with a protective immune reaction. Second, pathogens are not simple structures and their antigen composition is very complex and fluctuates widely. This feature is frequently underestimated as immunologists generally neglect the intra-species diversity of pathogens and their "within host evolution" during chronic infection (Pybus and Rambaut, 2009; Wilson, 2012). Small RNA viruses such as HIV are a welldocumented example of this. These viruses express a limited number of genes, but produce, within a very short period of time, an extraordinarily high number of variants in the 
host, thereby generating a dynamic heterogeneous population (termed quasi-species or cloud) with high antigen complexity (Domingo et al., 2012). Similarly, bacteria can display great diversity in the host, rapidly evolve within it (Shin et al., 2004; Hoboth et al., 2009) and follow a complex cycle (Justice et al., 2004). This structural complexity is conducive to the sharing of antigenic determinants between pathogens and the probability of cross-immune reactions. Lastly, in contrast to inbred naive mice, the variability of the adaptive immune response of individuals in nature is non negligible, even against simple proteins. This characteristic is notably due to the initial variability of the adaptive immune repertoire, as described in Oudin's seminal work on idiotypy in the rabbit in 1969 (Oudin and Michel, 1969), and to the individual's immune history which shapes the BCR and TCR repertoire. Thus, a large part of the apparent specificity of the adaptive immune response derives from the systematic use of highly reductionist experimental models selected by experimenters for their reproducibility and predictability. This conclusion should not be seen as a general challenge of the reductionist approach that is an inevitable stage in biological research, but as a reminder that our perception of reality results from the way in which we study it. We must constantly ask ourselves what we are neglecting in our models and whether or not we want to refine our understanding of reality, as this may affect our results.

From an evolutionary point of view, it would appear that the selection of an immune system displaying the potential to mediate cross-protective reactions is ineluctable to counter the selective pressure of rapidly adapting pathogens displaying complex escape immune mechanisms. Antigenic variation is one of the most common escape strategies of pathogens. The possibility of antigen-unspecific activation of immune effectors can potentially offer protection against new antigenic variants of pathogens. Numerous pathogens are also able to hide their antigens from the immune system and induce local anti-inflammatory environments. The Mackaness effect induced by unrelated infection can allow for the elimination or control of these stealth pathogens. Finally, the ability of the individual immune system to integrate and process all immune experiments results in a partially unpredictable immune response against infection. This may have also favored population survival as unpredictable individual responses promote the diversity of immune responses inside a population and thereby potential herd immunity to infection (discussed in Muraille, 2014). Thus, immune cross-reactions may not be a negligible "by-product" of the immune system response, but rather one of its fundamental traits in nature. As each individual initially produces a private $\mathrm{T}$ and $\mathrm{B}$ repertoire by random rearrangement of genes (reviewed in Muraille, 2014) and displays a particular immune history and a specific microbiota (Monteiro-da-Silva et al., 2014; Sato et al., 2015), cross-protection appears to be largely unpredictable at the individual level. However, we can reasonably expect that a better understanding of the underlying mechanisms will allow us to statistically predict crossprotection at the population level and integrate this phenomenon into future vaccination strategies. The field of nonspecific AIID is still in its infancy and needs to be systematically explored.

\section{PRACTICAL IMPLICATIONS AND PERSPECTIVES}

The paradigm of the antigen specificity of AIID has dominated the field of immunology for decades. The great majority of prophylactic and therapeutic immune treatments, such as subunit vaccines, antigen-specific immunotherapies against autoimmune diseases, tumors, and allergies, successfully exploit the specific side of acquired immunity. Acceptance of the nonspecific side of acquired immunity could have several important consequences on how we conceive self-tolerance, vaccination strategies and antitumoral immunity, and highlight the importance of considering the microbiota and chronic infectious pathogens as part of the host defense system against infection under natural conditions.

\section{Revisiting the Theory of Immune Tolerance}

If a natural infection were to lead to an increased frequency of lymphocytes displaying a large range of reactivities, crossreaction with a self-antigen would become frequent and inevitable. This would also mean that the pre-immune repertoire of experienced healthy individuals should display highaffinity antigen receptors specific for self-determinants. This phenomenon has been documented in healthy mice and humans (Avrameas et al., 2007; Nagele et al., 2013), but remains unexplained using the classical paradigms of tolerance. Classical self-tolerance theories propose that the choice of the immune system to tolerate or reject is based on the detection of a "simple" qualitative signal such as a microbial signature (stranger/pattern recognition theory; Janeway, 1989), damage signatures (danger theory; Matzinger, 2002), or more recently an abrupt discontinuity of the antigen signal (discontinuity theory; Pradeu et al., 2013) (Figure 2A). However, this approach does not take into consideration the connection between the components of the immune system and more particularly the highly interconnected network of the many innate and adaptive immune detectors. As discussed by Kitano (Kitano and Oda, 2006; Oda and Kitano, 2006), some parts of the immune network display a characteristic "bow tie" architecture (Figure 2B). Typically, this type of architecture detects a wide range of inputs through a degenerate detection system coupled to a "central processing unit" (conserved core), where the inputs are organized and processed. In turn, this system can provide a large variety of responses leading to non-trivial (unreliable, partially unpredictable) causal action (reviewed in Tieri et al., 2010). This suggests that immune tolerance could be the result of an elaborate computation by the immune network based on a very large set of parameters including microbial and damage signatures, but also a great number of other contextual parameters such as the location and duration of antigenic signals, the individual immune history and the general state of the host organism. In other terms, the immune network 


\section{A classical tolerance theories}

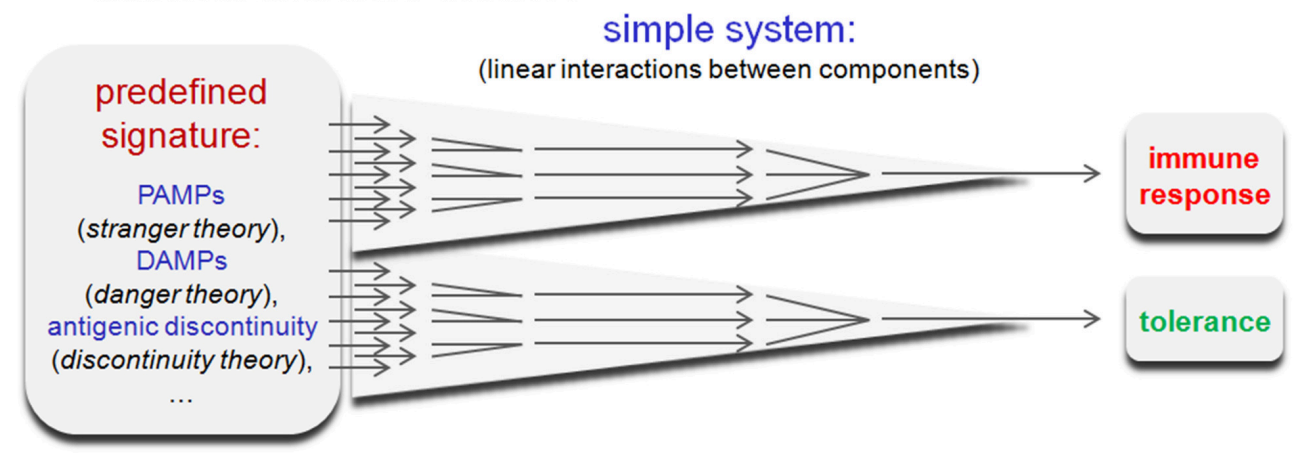

\section{B bow tie tolerance theory}

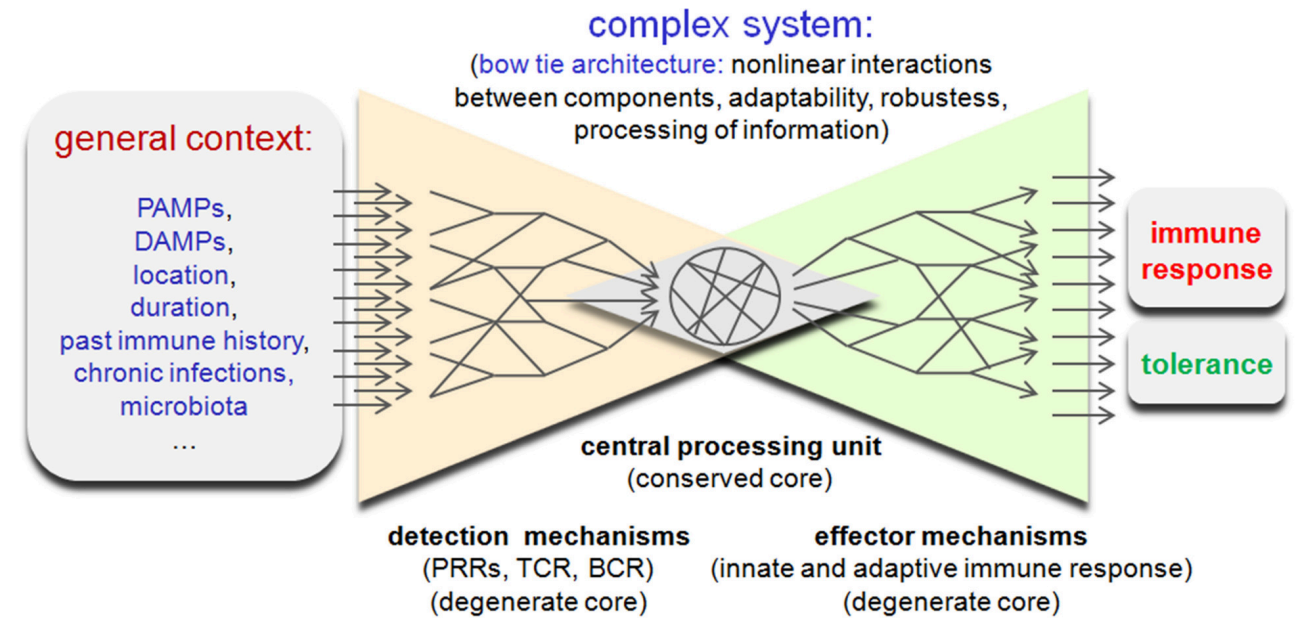

FIGURE 2 | Comparison of (A) "classical" and (B) "bow tie" tolerance theories.

could acts as a "cognition system," like the central nervous system, and be capable of information processing, learning, memorization, and adaptation. From this perspective, tolerance results from the interpretation of multiple signals in a general context. Of course, this does not mean that all signals have the same value. The immune system can focus on some signals, such as PAMPs or DAMPs, but the decision process remains dependant on the general context and requires information processing. This "bow tie hypothesis" fits with my proposal (Muraille, 2013) that the immune system was initially selected during evolution to allow for cooperation with the microbiota by tolerating cooperative and fighting selfish/cheater partners. It seems evident that endogenous (tumors) or exogenous (microbial pathogens) cheaters cannot be identified as such based only on a predefined signature, but on the evaluation of context-dependent behavior. This analysis requires an elaborate computation process and is indispensable to adapt to an evolving and unpredictable antigenic environment as well as to discriminate between noise and significant signals. In summary, the bow tie hypothesis suggests that a better understanding of tolerance can only come from a holistic approach to processing information networks within the immune system.
In contrast, previous theories on tolerance propose mainly to focus research on the identification of specific signals (PAMPs, DAMP, etc.) supposed to regulate the tolerance process directly (linearly).

\section{Vaccination Strategies}

Historically, the great success of vaccination campaigns has been associated with the administration of live-attenuated vaccines, such as Jenner's smallpox vaccine, the Bacillus Calmette-Guérin (BCG) vaccine, Sabin's polio vaccine and Hilleman's measles, mumps, and rubella vaccine. As previously discussed, these vaccines could display important non antigen-specific protective effects dependent on the innate or adaptive immune system. Thus, some of these vaccines could have important beneficial effects on populations even if their respective target diseases have been eliminated. For example, several epidemiological studies (Aaby et al., 2006; Jensen et al., 2006; Sørup et al., 2011) showed that smallpox-vaccinated individuals are at a lower risk of hospitalization for most subgroups of infectious diseases when compared to individuals not vaccinated for smallpox. As a result, it may be important to quantify the nonspecific beneficial effects associated with each vaccine before 
restricting their use. Modern vaccination strategies focus on the use of "adjuvanted" subunit vaccines (Pérez et al., 2013; Reed et al., 2013; De Gregorio and Rappuoli, 2014) that drive immune responses against dominant antigens expressed with low variability by pathogens. When compared to live-attenuated vaccines, there are several strong arguments in favor of subunit vaccines: (i) their supposed safety because they exclude all risks of reversion of attenuated pathogens to a virulent form, (ii) their high specificity limiting the risk of autoimmune diseases, and (iii) the difficulty to cultivate, conserve and transport live vaccines. However, the ability of adjuvanted subunit vaccines to induce trained immunity and cross-reactive lymphocytes is poorly documented. Modern synthetic adjuvants are first of all selected for their low inflammatory potential and it is not sure that they stimulate innate immunity that is strong enough and long enough to induce trained immunity. It could be interesting to systematically investigate the ability of synthetic adjuvants to induce trained immunity. Subunit vaccine strategies generally target a reduced number of antigenic structures and limit cross reactivity, thereby completely neglecting the adaptive capacity of pathogens. Indeed, microbial pathogens frequently display a very complex and fluctuating antigen composition. In addition to presenting important intra-species diversity, they show "within host evolution" during chronic infection (Pybus and Rambaut, 2009; Wilson, 2012). Small RNA viruses such as HIV are a well-documented example of this. These viruses express a limited number of genes but produce, in a very short period of time, an extraordinarily high number of variants in the host, generating a dynamic heterogeneous population (termed quasi-species or cloud) with high antigen complexity (Domingo et al., 2012). Similarly, bacteria can display great diversity in the host and rapidly evolve within it (Shin et al., 2004; Hoboth et al., 2009). A stable specific selective pressure is unable to control rapidly evolving pathogens for long. This could explain why subunit vaccine strategies have failed for decades to control numerous pandemics such as those associated with HIV, tuberculosis and malaria. In comparison, live vaccines display wide coverage against a multiplicity of antigens and are able to better cope with pathogen strain polymorphisms and host genetic restrictions. Thus, rational replacement of old vaccines with modern versions should be based on the assessment of both the specific and nonspecific benefits of each vaccine. A better understanding and exploitation of the crossprotection conferred by vaccines could open up new avenues in vaccine research, particularly in the development of multior poly-reactive vaccines. Along these lines, the World Health Organization recognized the importance of the nonspecific effects of vaccines in April 2014 and recommended further research in this new direction (World Health Organization, 2014).

\section{Manipulation of the Microbiota}

Modification of the microbiota composition by probiotics (Alexandre et al., 2014) and microbiota transfer (Austin et al., 2014) can constitute a powerful and not yet fully explored strategy to prevent or fight infection with multiresistant or highly-variable pathogens that escape classical antibiotic therapies or vaccination. For example, recent work on Plasmodium infection revealed that the host's microbiota plays an important role in the control of malaria (Yilmaz et al., 2014). The authors demonstrated that both Plasmodium spp. and the human gut pathobiont Escherichia coli O86:B7 express $\alpha$-gal, and that anti- $\alpha$-gal antibodies are associated with protection against malaria transmission in humans, suggesting that gut probiotics could represent innovative tools for malaria prevention. Thus, a single $\alpha$-gal displayed by a bacterium can affect resistance to a protozoon. This is yet another perfect demonstration that acquired resistance to infection can be nonspecific to the pathogen encountered. In the same way, therapeutic use of lytic bacteriophages (O'Flaherty et al., 2009) or predatory bacteria (Allen et al., 2014) to treat pathogenic bacterial infections is one approach that has great potential as a solution to the serious worldwide problem of drugresistant bacteria. In addition to offering an alternative solution to antibiotics, these therapies allow for more selective elimination of pathogenic bacteria without deeply affecting the host microbiota and reducing its resistance to opportunist infection.

\section{Nonspecific Immune Control of Tumors}

Despite considerable progress in cancer genetics, the vast majority of metastatic solid tumors still remain incurable today. It has become clear that tumors are heterogeneous structures that, during development and growth, become "sculpted" or "edited" by immune responses and, as a result, pass through the "three E's" of elimination, equilibration and escape (reviewed in Gerlinger et al., 2014). This Darwinian evolution of tumors within hosts explains the short-lived effects of immunotherapeutic strategies based on one single or a few tumor antigens and the difficulty to identify biomarkers common to large groups of tumors. Genetically distinct subpopulations of tumor cells have also been observed to cooperate to promote tumor growth, progression, and maintenance (Gerlinger et al., 2014). Interestingly, epidemiological studies have clearly established an inverse relationship between acute infections and cancer development (Rastogi et al., 2008; Krone et al., 2014). Spontaneous tumor regression has been observed in association with a wide range of infectious organisms including those of bacterial, fungal, viral, and protozoal origin (Reilly and Christine, 1953; Oliveira et al., 2001; Rosenberg et al., 2002; Baird et al., 2013). Not all types of microorganisms are expected to have the same anticarcinogenic effect; for example, viral infections seem to be mainly procarcinogenic, in contrast to bacteria or parasitic worms that more frequently exhibit antitumorigenic effects. In contrast to acute infections, chronic infections can be viewed as resulting from a failed immune response and an increasing number have been associated with an elevated risk of cancer (Hoption Cann et al., 2006). There are scores of ways in which infectious diseases can alter the course of cancers, and this should remain an area of intense study. Once again, in contrast to the classical specific approach focusing on the identification of specific tumor antigens, a promising focus of research could be 
based on exploitation of the nonspecific protective effect of the pathogen-induced immune response. Indeed, several experimental studies (reviewed in Bolhassani and Zahedifard, 2012) suggest that at least some pathogens and live vaccines may be beneficial with respect to the subsequent risk of cancer.

\section{REFERENCES}

Aaby, P., Benn, C., Nielsen, J., Lisse, I. M., Rodrigues, A., and Ravn, H. (2012). Testing the hypothesis that diphtheria-tetanus-pertussis vaccine has negative non-specific and sex-differential effects on child survival in high-mortality countries. BMJ Open 2:e000707. doi: 10.1136/bmjopen-2011-000707

Aaby, P., Gustafson, P., Roth, A., Rodrigues, A., Fernandes, M., Sodemann, M., et al. (2006). Vaccinia scars associated with better survival for adults. Vaccine 24, 5718-5725. doi: 10.1016/j.vaccine.2006.04.045

Aaby, P., Kollmann, T. R., and Benn, C. S. (2014). Nonspecific effects of neonatal and infant vaccination: public-health, immunological and conceptual challenges. Nat. Immunol. 15, 895-899. doi: 10.1038/ni.2961

Alberts, B., Johnson, A., Lewis, J., Raff, M., Roberts, K., and Walter, P. (2002). Molecular Biology of the Cell, 4th Edn. New York, NY: Garland Science.

Alexandre, Y., Le Blay, G., Boisramé-Gastrin, S., Le Gall, F., Héry-Arnaud, G., Gouriou, S., et al. (2014). Probiotics: a new way to fight bacterial pulmonary infections? Med. Mal. Infect. 44, 9-17. doi: 10.1016/j.medmal.2013.05.001

Allen, H. K., Trachsel, J., Looft, T., and Casey, T. A. (2014). Finding alternatives to antibiotics. Ann. N.Y. Acad. Sci. 1323, 91-100. doi: 10.1111/nyas.12468

Austin, M., Mellow, M., and Tierney, W. M. (2014). Fecal microbiota transplantation in the treatment of clostridium difficile infections. Am. J. Med. 127, 479-483. doi: 10.1016/j.amjmed.2014.02.017

Avrameas, S., Ternynck, T., Tsonis, I. A., and Lymberi, P. (2007). Naturally occurring B-cell autoreactivity: a critical overview. J. Autoimmun. 29, 213-218. doi: 10.1016/j.jaut.2007.07.010

Baird, J. R., Fox, B. A., Sanders, K. L., Lizotte, P. H., Cubillos-Ruiz, J. R., Scarlett, U. K., et al. (2013). Avirulent toxoplasma gondii generates therapeutic antitumor immunity by reversing immunosuppression in the ovarian cancer microenvironment. Cancer Res. 73, 3842-3851. doi: 10.1158/0008-5472.CAN$12-1974$

Barton, E. S., White, D. W., Cathelyn, J. S., Brett-McClellan, K. A., Engle, M., Diamond, M. S., et al. (2007). Herpesvirus latency confers symbiotic protection from bacterial infection. Nature 447, 326-329. doi: 10.1038/nature05762

Baumgarth, N. (2011). The double life of a B-1 cell: self-reactivity selects for protective effector functions. Nat. Rev. Immunol. 11, 34-46. doi: $10.1038 /$ nri2901

Belkaid, Y., Piccirillo, C. A., Mendez, S., Shevach, E. M., and Sacks, D. L. (2002). $\mathrm{CD} 4+\mathrm{CD} 25+$ regulatory $\mathrm{T}$ cells control Leishmania major persistence and immunity. Nature 420, 502-507. doi: 10.1038/nature01152

Benn, C. S., Netea, M. G., Selin, L. K., and Aaby, P. (2013). A small jab - a big effect: nonspecific immunomodulation by vaccines. Trends Immunol. 34, 431-439. doi: 10.1016/j.it.2013.04.004

Bernasconi, N. L., Traggiai, E., and Lanzavecchia, A. (2002). Maintenance of serological memory by polyclonal activation of human memory B cells. Science 298, 2199-2202. doi: 10.1126/science.1076071

Beura, L. K., Anderson, K. G., Schenkel, J. M., Locquiao, J. J., Fraser, K. A., Vezys, V., et al. (2015). Lymphocytic choriomeningitis virus persistence promotes effector-like memory differentiation and enhances mucosal T cell distribution. J. Leukoc. Biol. 97, 217-225. doi: 10.1189/jlb.1HI0314-154R

Blanden, R. V., Lefford, M. J., and Mackaness, G. B. (1969). The host response to Calmette-Guérin bacillus infection in mice. J. Exp. Med. 129, 1079-1107. doi: 10.1084/jem.129.5.1079

Blok, B. A., Arts, R. J. W., van Crevel, R., Benn, C. S., and Netea, M. G. (2015). Trained innate immunity as underlying mechanism for the longterm, nonspecific effects of vaccines. J. Leukoc. Biol. 98, 347-356. doi: 10.1189/jlb.5RI0315-096R

\section{ACKNOWLEDGMENTS}

EM is a Research Associate with the Fonds National de la Recherche Scientifique (FNRS), Belgium. The author wishes to thank Jean-Jacques Letesson, Oberdan Leo and Carl De Trez for their helpful advice and discussions.

Bolhassani, A., and Zahedifard, F. (2012). Therapeutic live vaccines as a potential anticancer strategy. Int. J. Cancer 131, 1733-1743. doi: 10.1002/ijc.27640

Bonneville, M., O’Brien, R. L., and Born, W. K. (2010). Gammadelta T cell effector functions: a blend of innate programming and acquired plasticity. Nat. Rev. Immunol. 10, 467-478. doi: 10.1038/nri2781

Bosch, A. A. T. M., Biesbroek, G., Trzcinski, K., Sanders, E. A. M., and Bogaert, D. (2013). Viral and bacterial interactions in the upper respiratory tract. PLoS Pathog. 9:e1003057. doi: 10.1371/journal.ppat.1003057

Bosch, T. C. G., and McFall-Ngai, M. J. (2011). Metaorganisms as the new frontier. Zool Jena Ger 114, 185-190. doi: 10.1016/j.zool.2011.04.001

Brownlee, J. (2007). Complex adaptive systems. CIS Technol. Rep. 32, 183-204. doi: 10.1146/annurev.anthro.32.061002.093440

Buffie, C. G., and Pamer, E. G. (2013). Microbiota-mediated colonization resistance against intestinal pathogens. Nat. Rev. Immunol. 11, 790-801. doi: $10.1038 /$ nri3535

Cassat, J. E., and Skaar, E. P. (2013). Iron in infection and immunity. Cell Host Microbe 13, 509-519. doi: 10.1016/j.chom.2013.04.010

Cerf-Bensussan, N., and Gaboriau-Routhiau, V. (2010). The immune system and the gut microbiota: friends or foes? Nat. Rev. Immunol. 10, 735-744. doi: $10.1038 /$ nri2850

Chen, Z. J., Wheeler, C. J., Shi, W., Wu, A. J., Yarboro, C. H., Gallagher, M., et al. (1998). Polyreactive antigen-binding B cells are the predominant cell type in the newborn B cell repertoire. Eur. J. Immunol. 28, 989-994. doi: 10.1002/(SICI)1521-4141(199803)28:03 < 989::AID-IMMU989>3.0.CO;2-1

Cooper, M. A., Elliott, J. M., Keyel, P. A., Yang, L., Carrero, J. A., and Yokoyama, W. M. (2009). Cytokine-induced memory-like natural killer cells. Proc. Natl. Acad. Sci. U.S.A. 106, 1915-1919. doi: 10.1073/pnas.0813192106

Curtsinger, J. M., Lins, D. C., and Mescher, M. F. (1998). CD8+ memory T cells (CD44high, Ly-6C+) are more sensitive than naive cells to (CD44low, Ly-6C-) to TCR/CD8 signaling in response to antigen. J. Immunol. 160, 3236-3243.

De Gregorio, E., and Rappuoli, R. (2014). From empiricism to rational design: a personal perspective of the evolution of vaccine development. Nat. Rev. Immunol. 14, 505-14. doi: 10.1038/nri3694

Degli-Esposti, M. A., and Smyth, M. J. (2005). Close encounters of different kinds: dendritic cells and NK cells take centre stage. Nat. Rev. Immunol. 5, 112-124. doi: $10.1038 /$ nri1549

Didierlaurent, A., Goulding, J., and Hussell, T. (2007). The impact of successive infections on the lung microenvironment. Immunology 122, 457-465. doi: 10.1111/j.1365-2567.2007.02729.x

Domingo, E., Sheldon, J., and Perales, C. (2012). Viral quasispecies evolution. Microbiol. Mol. Biol. Rev. 76, 159-216. doi: 10.1128/MMBR.05023-11

Eberl, G. (2010). A new vision of immunity: homeostasis of the superorganism. Mucosal Immunol. 3, 450-460. doi: 10.1038/mi.2010.20

Eisen, H. N., and Chakraborty, A. K. (2010). Evolving concepts of specificity in immune reactions. Proc. Natl. Acad. Sci. U.S.A. 107, 22373-22380. doi: 10.1073/pnas. 1012051108

Fazekas de St Groth and Webster, R. G. (1966). Disquisitions on original antigenic sin. II. proof in lower creatures. J. Exp. Med. 124, 347-361. doi: 10.1084/jem.124.3.347

Foo, S. Y., and Phipps, S. (2010). Regulation of inducible BALT formation and contribution to immunity and pathology. Mucosal Immunol. 3, 537-544. doi: $10.1038 / \mathrm{mi} .2010 .52$

Frenkel, J. K., and Caldwell, S. A. (1975). Specific immunity and nonspecific resistance to infection: listeria, protozoa, and viroses in mice and hamsters. J. Infect. Dis. 131, 201-209. doi: 10.1093/infdis/131.3.201 
Fu, Z. Q., and Dong, X. (2013). Systemic acquired resistance: turning local infection into global defense. Annu. Rev. Plant Biol. 64, 839-863. doi: 10.1146/annurev-arplant-042811-105606

Furman, D., Jojic, V., Sharma, S., Shen-orr, S. S., Angel, C. J. L., Onengut-gumuscu, S., et al. (2015). Cytomegalovirus infection enhances the immune response to influenza. Sci. Transl. Med. 7:281ra43. doi: 10.1126/scitranslmed.aaa2293

Gerlinger, M., McGranahan, N., Dewhurst, S. M., Burrell, R. A., Tomlinson, I., and Swanton, C. (2014). Cancer: evolution within a lifetime. Annu. Rev. Genet. 48, 215-238. doi: 10.1146/annurev-genet-120213-092314

Hoboth, C., Hoffmann, R., Eichner, A., Henke, C., Schmoldt, S., Imhof, A., et al. (2009). Dynamics of adaptive microevolution of hypermutable Pseudomonas aeruginosa during chronic pulmonary infection in patients with cystic fibrosis. J. Infect. Dis. 200, 118-130. doi: 10.1086/599360

Holland, J. H. (2006). Studying complex adaptive systems. J. Syst. Sci. Complex 19, 1-8. doi: 10.1007/s11424-006-0001-Z

Hoption Cann, S. A., Van Netten, J. P., and Van Netten, C. (2006). Acute infections as a means of cancer prevention: opposing effects to chronic infections? Cancer Detect. Prev. 30, 83-93. doi: 10.1016/j.cdp.2005.11.001

Ivanov, I. I., Atarashi, K., Manel, N., Brodie, E. L., Shima, T., Karaoz, U., et al. (2009). Induction of intestinal Th17 cells by segmented filamentous bacteria. Cell 139, 485-498. doi: 10.1016/j.cell.2009.09.033

Janeway, C. A. (1989). Approaching the asymptote? Evolution and revolution in immunology. Cold Spring Harb. Symp. Quant. Biol. 54(Pt 1), 1-13. doi: 10.1101/SQB.1989.054.01.003

Jayasekera, J. P., Moseman, E. A., and Carroll, M. C. (2007). Natural antibody and complement mediate neutralization of influenza virus in the absence of prior immunity. J. Virol. 81, 3487-3494. doi: 10.1128/JVI.02128-06

Jensen, M. L., Dave, S., van der Loeff, M. S., da Costa, C., Vincent, T., Leligdowicz, A., et al. (2006). Vaccinia scars associated with improved survival among adults in rural guinea-bissau. PLoS ONE 1:e101. doi: 10.1371/journal.pone.0000101

Joshi, S. K., Suresh, P. R., and Chauhan, V. S. (2001). Flexibility in MHC and TCR recognition: degenerate specificity at the $\mathrm{T}$ cell level in the recognition of promiscuous Th epitopes exhibiting no primary sequence homology. J. Immunol. 166, 6693-6703. doi: 10.4049/jimmunol.166.11.6693

Justice, S. S., Hung, C., Theriot, J. A., Fletcher, D. A., Anderson, G. G., Footer, M. J., et al. (2004). Differentiation and developmental pathways of uropathogenic Escherichia coli in urinary tract pathogenesis. Proc. Natl. Acad. Sci. U.S.A. 101, 1333-1338. doi: 10.1073/pnas.0308125100

Kamada, N., Chen, G. Y., Inohara, N., and Neúñz, G. (2013). Control of pathogens and pathobionts by the gut microbiota. Nat. Immunol. 14, 685-690. doi: 10.1038/ni.2608

Keppel, M. P., Yang, L., and Cooper, M. A. (2013). Murine NK cell intrinsic cytokine-induced memory-like responses are maintained following homeostatic proliferation. J. Immunol. 190, 4754-4762. doi: 10.4049/jimmunol.1201742

Kitano, H., and Oda, K. (2006). Robustness trade-offs and host-microbial symbiosis in the immune system. Mol. Syst. Biol. 2, 2006.0022. doi: $10.1038 / \mathrm{msb} 4100039$

Kleinnijenhuis, J., Quintin, J., Preijers, F., Joosten, L. A. B., Ifrim, D. C., Saeed, S., et al. (2012). Bacille calmette-guerin induces NOD2-dependent nonspecific protection from reinfection via epigenetic reprogramming of monocytes. Proc. Natl. Acad. Sci. U.S.A. 109, 17537-17542. doi: 10.1073/pnas.1202870109

Krone, B., Kölmel, K. F., and Grange, J. M. (2014). The biography of the immune system and the control of cancer: from St Peregrine to contemporary vaccination strategies. BMC Cancer 14:595. doi: 10.1186/1471-2407-14-595

Mackaness, G. B. (1964). The immunological basis of acquired cellular resistance. J. Exp. Med. 120, 105-120. doi: 10.1084/jem.120.1.105

Martin, S. F. (2014). Adaptation in the innate immune system and heterologous innate immunity. Cell. Mol. Life Sci. 21, 4115-4130. doi: 10.1007/s00018-0141676-2

Matsumoto, S., Yukitake, H., Kanbara, H., Yamada, H., Kitamura, A., and Yamada, T. (2000). Mycobacterium bovis bacillus calmette-guérin induces protective immunity against infection by Plasmodium yoelii at blood-stage depending on shifting immunity toward Th1 type and inducing protective IgG2a after the parasite infection. Vaccine 19, 779-787. doi: 10.1016/S0264-410X(00) 00257-7

Matzinger, P. (2002). The danger model: a renewed sense of self. Science 296, 301-305. doi: 10.1126/science.1071059
Mayr, A. (2004). Taking advantage of the positive side-effects of smallpox vaccination. J. Vet. Med. B Infect. Dis. Vet. Public Health 51, 199-201. doi: 10.1111/j.1439-0450.2004.00763.x

Mina, M. J., Metcalf, C. J., de Swart, R. L., Osterhaus, A., and Grenfell, B. (2015). Long-term measles-induced immunomodulation increases overall childhood infectious disease mortality. Science 348, 694-699. doi: 10.1126/science.aaa3662

Min-Oo, G., Kamimura, Y., Hendricks, D. W., Nabekura, T., and Lanier, L. L. (2013). Natural killer cells: walking three paths down memory lane. Trends Immunol. 34, 251-258. doi: 10.1016/j.it.2013.02.005

Monteiro-da-Silva, F., Araujo, R., and Sampaio-Maia, B. (2014). Interindividual variability and intraindividual stability of oral fungal microbiota over time. Med. Mycol. 52, 496-503. doi: 10.1093/mmy/myu027

Mueller, S. N., and Germain, R. N. (2009). Stromal cell contributions to the homeostasis and functionality of the immune system. Nat. Rev. Immunol. 9, 618-629. doi: 10.1038/nri2588

Muraille, E. (2013). Redefining the immune system as a social interface for cooperative processes. PLoS Pathog. 9:e1003203. doi: 10.1371/journal.ppat.1003203

Muraille, E. (2014). Generation of individual diversity: a too neglected fundamental property of adaptive immune system. Front. Immunol. 5:208. doi: 10.3389/fimmu.2014.00208

Murphy, K., Travers, P., and Walport, M. (2008). Janeway's Immunobiology. New York, NY: Garland Science.

Nagele, E. P., Han, M., Acharya, N. K., DeMarshall, C., Kosciuk, M. C., and Nagele, R. G. (2013). Natural IgG autoantibodies are abundant and ubiquitous in human sera, and their number is influenced by age, gender, and disease. PLoS ONE 8:e60726. doi: 10.1371/journal.pone.0060726

Nairz, M., Schroll, A., Sonnweber, T., and Weiss, G. (2010). The struggle for iron - a metal at the host-pathogen interface. Cell. Microbiol. 12, 1691-1702. doi: 10.1111/j.1462-5822.2010.01529.x

Netea, M. G., Quintin, J., and van der Meer, J. W. M. (2011). Trained immunity: a memory for innate host defense. Cell Host Microbe 9, 355-361. doi: 10.1016/j.chom.2011.04.006

Notkins, A. L. (2004). Polyreactivity of antibody molecules. Trends Immunol. 25, 174-179. doi: 10.1016/j.it.2004.02.004

O'Flaherty, S., Ross, R. P., and Coffey, A. (2009). Bacteriophage and their lysins for elimination of infectious bacteria: review article. FEMS Microbiol. Rev. 33, 801-819. doi: 10.1111/j.1574-6976.2009.00176.x

Obar, J. J., Fuse, S., Leung, E. K., Bellfy, S. C., and Usherwood, E. J. (2006). Gammaherpesvirus persistence alters key CD8 T-cell memory characteristics and enhances antiviral protection. J. Virol. 80, 8303-8315. doi: 10.1128/JVI.00237-06

Ochsenbein, A. F., Fehr, T., Lutz, C., Suter, M., Brombacher, F., Hengartner, H., et al. (1999). Control of early viral and bacterial distribution and disease by natural antibodies. Science 286, 2156-2159. doi: 10.1126/science.286.5447.2156

Oda, K., and Kitano, H. (2006). A comprehensive map of the toll-like receptor signaling network. Mol. Syst. Biol. 2, 2006.0015. doi: 10.1038/msb4100057

Oikonomopoulou, K., Brinc, D., Kyriacou, K., and Diamandis, E. P. (2013). Infection and cancer: revaluation of the hygiene hypothesis. Clin. Cancer Res. 19, 2834-2841. doi: 10.1158/1078-0432.CCR-12-3661

Oldstone, M. B. (2014). Molecular mimicry: its evolution from concept to mechanism as a cause of autoimmune diseases. Monoclon. Antib. Immunodiagn. Immunother. 33, 158-165. doi: 10.1089/mab.2013.0090

Oliveira, E. C., Leite, M. S., Miranda, J. A., Andrade, A. L., Garcia, S. B., Luquetti, A. O., et al. (2001). Chronic Trypanosoma cruzi infection associated with low incidence of 1,2-dimethylhydrazine-induced colon cancer in rats. Carcinogenesis 22, 737-740. doi: 10.1093/carcin/22.5.737

Oudin, J., and Michel, M. (1969). Idiotypy of rabbit antibodies. I. Comparison of idiotypy of antibodies against Salmonella typhi with that of antibodies against other bacteria in the same rabbits, or of antibodies against Salmonella typhi in various rabbits. J. Exp. Med. 1956, 595-617. doi: 10.1084/jem.130.3.595

Parra, M., Liu, X., Derrick, S. C., Yang, A., Tian, J., Kolibab, K., et al. (2013). Molecular analysis of non-specific protection against murine malaria induced by BCG vaccination. PLoS ONE 8:e66115. doi: 10.1371/journal.pone.0066115

Pérez, O., Romeu, B., Cabrera, O., González, E., Batista-Duharte, A., Labrada, A., et al. (2013). Adjuvants are key factors for the development of future vaccines: lessons from the finlay adjuvant platform. Front. Immunol. 4:407. doi: 10.3389/fimmu.2013.00407 
Perry, S., de Jong, B. C., Solnick, J. V., De La Luz Sanchez, M., Yang, S., Lin, P. L., et al. (2010). Infection with Helicobacter pylori is associated with protection against tuberculosis. PLoS ONE 5:e8804. doi: 10.1371/journal.pone.0008804

Potian, J. A., Rafi, W., Bhatt, K., McBride, A., Gause, W. C., and Salgame, P. (2011). Preexisting helminth infection induces inhibition of innate pulmonary antituberculosis defense by engaging the IL-4 receptor pathway. J. Exp. Med. 208, 1863-1874. doi: 10.1084/jem.20091473

Pradeu, T., Jaeger, S., and Vivier, E. (2013). The speed of change: towards a discontinuity theory of immunity? Nat. Rev. Immunol. 13, 764-769. doi: 10.1038/nri3521

Pybus, O. G., and Rambaut, A. (2009). Evolutionary analysis of the dynamics of viral infectious disease. Nat. Rev. Genet. 10, 540-550. doi: 10.1038/nrg2583

Quintin, J., Saeed, S., Martens, J. H. A., Giamarellos-Bourboulis, E. J., Ifrim, D. C., Logie, C., et al. (2012). Candida albicans infection affords protection against reinfection via functional reprogramming of monocytes. Cell Host Microbe 12, 223-232. doi: 10.1016/j.chom.2012.06.006

Rastogi, T., Devesa, S., Mangtani, P., Mathew, A., Cooper, N., Kao, R., et al. (2008). Cancer incidence rates among South Asians in four geographic regions: India, Singapore UK and US. Int. J. Epidemiol. 37, 147-160. doi: 10.1093/ije/dym219

Rawlings, D. J., Schwartz, M. A., Jackson, S. W., and Meyer-Bahlburg, A. (2012). Integration of B cell responses through Toll-like receptors and antigen receptors. Nat. Rev. Immunol. 12, 282-294. doi: 10.1038/nri3190

Reed, S. G., Orr, M. T., and Fox, C. B. (2013). Key roles of adjuvants in modern vaccines. Nat. Med. 19, 1597-1608. doi: 10.1038/nm.3409

Reilly, H. C., and Christine, H. (1953). Microbiology and cancer therapy: a review microbiology and cancer therapy: a review. Cancer Res. 13, 821-834.

Richer, M. J., Pewe, L. L., Hancox, L. S., Hartwig, S. M., Varga, S. M., and Harty, J. T. (2015). Inflammatory IL-15 is required for optimal memory T cell responses. J. Clin. Invest. 125, 1-14. doi: 10.1172/jci81261

Rosenberg, S. A., Spiess, P. J., and Kleiner, D. E. (2002). Antitumor effects in mice of the intravenous injection of attenuated Salmonella typhimurium. J. Immunother. 25, 218-225. doi: 10.1097/00002371-200205000-00004

Rossjohn, J., Pellicci, D. G., Patel, O., Gapin, L., and Godfrey, D. I. (2012). Recognition of CD1d-restricted antigens by natural killer T cells. Nat. Rev. Immunol. 12, 845-857. doi: 10.1038/nri3328

Sato, Y., Yamagishi, J., Yamashita, R., Shinozaki, N., Ye, B., Yamada, T., et al. (2015). Inter-individual differences in the oral bacteriome are greater than intra-day fluctuations in individuals. PLOS ONE 10:e131607. doi: 10.1371/journal.pone.0131607

Selin, L. K., Nahill, S. R., and Welsh, R. M. (1994). Cross-reactivities in memory cytotoxic T lymphocyte recognition of heterologous viruses. J. Exp. Med. 179, 1933-1943. doi: 10.1084/jem.179.6.1933

Selin, L. K., Wlodarczyk, M. F., Kraft, A. R., Nie, S., Kenney, L. L., Puzone, R., et al. (2011). Heterologous immunity: immunopathology, autoimmunity and protection during viral infections. Autoimmunity 44, 328-347. doi: 10.3109/08916934.2011.523277

Sewell, A. K. (2012). Why must T cells be cross-reactive? Nat. Rev. Immunol. 12, 669-677. doi: 10.1038/nri3279

Sher, N. A., Chaparas, S. D., Greenberg, L. E., and Bernard, S. (1975). Effects of BCG, Corynebacterium parvum, and methanol-extration residue in the reduction of mortality from Staphylococcus aureus and Candida albicans infections in immunosuppressed mice. Infect. Immun. 12, 1325-1330.

Shin, J. H., Park, M. R., Song, J. W., Shin, D. H., Jung, S. I., Cho, D., et al. (2004). Microevolution of Candida albicans strains during catheter-related candidemia. J. Clin. Microbiol. 42, 4025-4031. doi: 10.1128/JCM.42.9.40254031.2004

Sørup, S., Villumsen, M., Ravn, H., Benn, C. S., Sørensen, T. I., Aaby, P., et al. (2011). Smallpox vaccination and all-cause infectious disease hospitalization: a Danish register-based cohort study. Int. J. Epidemiol. 40, 955-963. doi: 10.1093/ije/dyr063

Soudja, S. M. H., Ruiz, A. L., Marie, J. C., and Lauvau, G. (2012). Inflammatory monocytes activate memory CD8+ T and innate NK lymphocytes independent of cognate antigen during microbial pathogen invasion. Immunity 37, 549-562. doi: 10.1016/j.immuni.2012.05.029

Stelekati, E., and Wherry, E. J. (2012). Chronic bystander infections and immunity to unrelated antigens. Cell Host Microbe 12, 458-469. doi: 10.1016/j.chom.2012.10.001

Strachan, D. P. (1989). Hay fever, hygiene, and household size. BMJ 299, 1259-1260. doi: 10.1136/bmj.299.6710.1259

Tanaka, K., Sawamura, S., Satoh, T., Kobayashi, K., and Noda, S. (2007). Role of the indigenous microbiota in maintaining the virus-specific CD8 memory $\mathrm{T}$ cells in the lung of mice infected with murine cytomegalovirus. J. Immunol. 178, 5209-5216. doi: 10.4049/jimmunol.178.8.5209

Tieri, P., Grignolio, A., Zaikin, A., Mishto, M., Remondini, D., Castellani, G. C., et al. (2010). Network, degeneracy and bow tie integrating paradigms and architectures to grasp the complexity of the immune system. Theor. Biol. Med. Model. 7:32. doi: 10.1186/1742-4682-7-32

Tribouley, J., Tribouley-Duret, J., and Appriou, M. (1978). Effect of bacillus callmette guerin (BCG) on the receptivity of nude mice to Schistosoma mansoni. C. R. Seances Soc. Biol. Fil. 172, 902-904.

Van't Wout, J. W., Poell, R., and Van Furth, R. (1992). The role of BCG/PPDactivated macrophages in resistance against systemic candidiasis in mice. Scand. J. Immunol. 36, 713-719. doi: 10.1111/j.1365-3083.1992.tb03132.x

Wang, J., Li, F., Sun, R., Gao, X., Wei, H., Li, L.-J., et al. (2013). Bacterial colonization dampens influenza-mediated acute lung injury via induction of M2 alveolar macrophages. Nat. Commun. 4, 2106. doi: 10.1038/ncomms3106

Welsh, R. M., and Selin, L. K. (2002). No one is naive: the significance of heterologous T-cell immunity. Nat. Rev. Immunol. 2, 417-426. doi: $10.1038 /$ nri820

Wilson, D. J. (2012). Insights from genomics into bacterial pathogen populations. PLoS Pathog. 8:e1002874. doi: 10.1371/journal.ppat.1002874

Wooldridge, L., Ekeruche-Makinde, J., Van den Berg, H. A., Skowera, A., Miles, J. J., Tan, M. P., et al. (2012). A single autoimmune T cell receptor recognizes more than a million different peptides. J. Biol. Chem. 287, 1168-1177. doi: 10.1074/jbc.M111.289488

World Health Organization (2014). Meeting of the strategic advisory group of experts on immunization, April 2014-conclusions and recommendations. Wkly. Epidemiol. Rec. 89, 221-236.

Wucherpfennig, K. W. (2004). T cell receptor crossreactivity as a general property of $\mathrm{T}$ cell recognition. Mol. Immunol. 40, 1009-1017. doi: 10.1016/j.molimm.2003.11.003

Wucherpfennig, K. W., Allen, P. M., Celada, F., Cohen, I. R., De Boer, R., Garcia, K. C., et al. (2007). Polyspecificity of T cell and B cell receptor recognition. Semin. Immunol. 19, 216-224. doi: 10.1016/j.smim.2007.02.012

Yilmaz, B., Portugal, S., Tran, T. M., Gozzelino, R., Ramos, S., Gomes, J., et al. (2014). Gut microbiota elicits a protective immune response against malaria transmission. Cell 159, 1277-1289. doi: 10.1016/j.cell.2014.10.053

Zarnitsyna, V. I., Evavold, B. D., Schoettle, L. N., Blattman, J. N., and Antia, R. (2013). Estimating the diversity, completeness, and cross-reactivity of the T cell repertoire. Front. Immunol. 4:485. doi: 10.3389/fimmu.2013.00485

Zola, T. A., Lysenko, E. S., and Weiser, J. N. (2009). Natural antibody to conserved targets of Haemophilus influenzae limits colonization of the murine nasopharynx. Infect. Immun. 77, 3458-3465. doi: 10.1128/IAI. 01564-08

Conflict of Interest Statement: The author declares that the research was conducted in the absence of any commercial or financial relationships that could be construed as a potential conflict of interest.

Copyright (C) 2016 Muraille. This is an open-access article distributed under the terms of the Creative Commons Attribution License (CC BY). The use, distribution or reproduction in other forums is permitted, provided the original author(s) or licensor are credited and that the original publication in this journal is cited, in accordance with accepted academic practice. No use, distribution or reproduction is permitted which does not comply with these terms. 